\title{
Particle swarm optimization and method of moments for modeling and optimization of microstrip antennas
}

\author{
Tarek Fortaki and Mounir Amir \\ Electronics Department \\ University of Batna \\ Batna, Algeria \\ t_fortaki@yahoo.fr \\ Siham Benkouda and Abdelkrim Belhedri \\ Electronics Department \\ University of Frères Mentouri - Constantine 1 \\ Constantine, Algeria \\ s_benkouda@yahoo.fr
}

\begin{abstract}
This paper introduces a novel technique for efficiently combining Particle Swarm Optimization (PSO) with method of moments (MOM) for computing the resonant frequency and bandwidth of rectangular microstrip antenna. In this technique, the problem is formulated in terms of an integral equation which is the kernel of a dyadic Green's function. After this PSO which will be introduce to determinate antenna parameters by optimizing the impedance matrix $|\mathrm{Z}|$. The resonant frequency results obtained by using (PSO/MOM) algorithm are in very good agreement with the experimental results available in the literature. The computation time is greatly reduced as compared to the classical MOM.
\end{abstract}

Keywords-component—particle swarm optimization (PSO); method of moments (MOM); microstrip antenna; modeling; optimization.

\section{INTRODUCTION}

Due to their many attractive features, microstrip antennas have drawn the attention of researchers over the past decades. Microstrip antennas are used in an increasing number of applications, ranging from biomedical diagnosis to wireless communication. Research on microstrip antenna in the 21 st aims at size reduction, high gain, resonant frequency, wide bandwidth, multiple functionality, and system-level integration [1]. Several methods are available in the literature for computing the resonant frequency of patch antennas. These methods can generally be divided into two groups: simple analytical methods and rigorous numerical methods. For rigorous methods like the moment method (MOM) the exact mathematical formulations involve extensive numerical procedures. This technique need an important time for calculation. A new methods the evolutionary algorithms, in particular the genetic algorithms (GA), have been widely used in electromagnetic applications in the last years [2]. More recently, a new stochastic optimization technique has rapidly gained popularity in the Electromagnetic Community: the Particle Swarm Optimization (PSO). PSO can be understood as a modeling via an analogy similar to the social activities of a bird flock, or a bee swarm. The PSO is a powerful technique that greatly simplifies the optimization process compared to ANN and
GA [3].In this paper, the combination of Particle Swarm Optimization (PSO) and moment method (MOM) is presented for the calculation of the resonant frequency and bandwidth. This combination is intended to reduce the computation time, and at the same time keep the quality of the results obtained by the moment method. The same algorithm can be also used for the optimization of geometrical parameters of microstrip antennas.

\section{ANALYSIS METHOD}

The problem to be solved is illustrated in figure 1 . We have a rectangular patch antenna with dimensions $(a \times b)$, is printed on a dielectric substrate isotropic of thickness $d$, is characterized by the free space permeability $\mu_{0}$ and a permittivity $\varepsilon$. The ambient medium is air with constitutive parameters $\mu_{0}$ and $\varepsilon_{0}$. Assuming an $\boldsymbol{e}^{i \omega t}$ time variations and starting from Maxwell's equations in the Fourier transform domain, we can show that the transverse fields inside the $\mathrm{j}$ layer $\left(\mathrm{Z}_{\mathrm{j}-1}<\mathrm{Z}<\mathrm{Z}_{\mathrm{j}}\right)$ can be written in terms of the longitudinal components $\tilde{E}_{z}$ and $\tilde{H}_{z}$ as [4-5]. 


$$
\tilde{\mathbf{E}}\left(k_{s}, z\right)=\left[\begin{array}{c}
\tilde{E}_{x}\left(k_{s}, z\right) \\
\tilde{E}_{y}\left(k_{s}, z\right)
\end{array}\right]=\overline{\mathbf{F}}\left(k_{s}\right) \cdot\left[\begin{array}{l}
\frac{1}{k_{s}} \frac{\partial \tilde{E}_{z}\left(k_{s}, z\right)}{\partial z} \\
\frac{\omega \mu_{0}}{k_{s}} \tilde{H}_{z}\left(k_{s}, z\right)
\end{array}\right]
$$

$$
\tilde{\mathbf{E}}\left(k_{s}, z\right)=\overline{\mathbf{F}}\left(k_{s}\right) \cdot \mathbf{e}\left(k_{s}, z\right)
$$

$$
\tilde{\mathbf{H}}\left(k_{s}, z\right)=\left[\begin{array}{c}
\tilde{H}_{y}\left(k_{s}, z\right) \\
-\tilde{H}_{x}\left(k_{s}, z\right)
\end{array}\right]=\overline{\mathbf{F}}\left(k_{s}\right) \cdot\left[\begin{array}{l}
\frac{\omega \varepsilon_{j}}{k_{s}} \tilde{H}_{z}\left(k_{s}, z\right) \\
\frac{1}{k_{s}} \frac{\partial \tilde{H}_{z}\left(k_{s}, z\right)}{\partial z}
\end{array}\right]
$$

$$
\tilde{\mathbf{H}}\left(k_{s}, z\right)=\overline{\mathbf{F}}\left(k_{s}\right) \cdot \mathbf{h}\left(k_{s}, z\right)
$$

Were $\mathrm{e}$ and $\mathrm{h}$ are, respectively, the transverse electric and magnetic fields in the (TM, TE) representation, and

$$
\bar{F}\left(k_{s}\right)=\frac{1}{k_{s}}\left[\begin{array}{cc}
k_{x} & k_{y} \\
k_{y} & -k_{x}
\end{array}\right]
$$

With $: k_{s}^{2}=k_{x}^{2}+k_{y}^{2}$

Substituting the expressions of $\tilde{E}_{z}$ and $\tilde{H}_{z}$ [4-6] into (1) and (2), we get

$$
\begin{aligned}
& \tilde{E}_{z}=A \cdot e^{-i k_{Z} Z}+B \cdot e^{i k_{z} Z} \\
& \tilde{H}_{z}=\bar{g}\left(k_{s}\right) \cdot\left[A \cdot e^{-i k_{z} Z}-B \cdot e^{i k_{Z} Z}\right]
\end{aligned}
$$

In (4) and (5), A and B are two-component unknown vectors and

$$
\overline{\mathbf{g}}\left(k_{s}\right)=\operatorname{diag}\left[\frac{\omega \varepsilon}{k_{z}}, \frac{k_{z}}{\omega \mu}\right]
$$

Writing (4) and (5) in the planes $z=z_{j-1}$ and $z=z_{j}$, and by eliminating the unknowns $\mathrm{A}$ and $\mathrm{B}$, we obtain the matrix form

$$
\left[\begin{array}{c}
\mathbf{e}\left(k_{s}, z_{j}^{-}\right) \\
\mathbf{h}\left(k_{s}, z_{j}^{-}\right)
\end{array}\right]=\overline{\mathbf{T}}_{j} \cdot\left[\begin{array}{c}
\mathbf{e}\left(k_{s}, z_{j-1}^{+}\right) \\
\mathbf{h}\left(k_{s}, z_{j-1}^{+}\right)
\end{array}\right]
$$

$$
\begin{array}{r}
\overline{\mathbf{T}}_{j}=\left[\begin{array}{cc}
\overline{\mathbf{T}}_{j}^{11} & \overline{\mathbf{T}}_{j}^{12} \\
\overline{\mathbf{T}}_{j}^{21} & \overline{\mathbf{T}}_{j}^{22}
\end{array}\right] \\
\overline{\mathbf{T}}_{j}=\left[\begin{array}{cc}
\cos \left(k_{z j} \cdot d_{j}\right) & -i \cdot \overline{\mathbf{g}}^{-1} \cdot \sin \left(k_{z j} \cdot d_{j}\right) \\
-i \cdot \overline{\mathbf{g}} \cdot \sin \left(k_{z j} \cdot d_{j}\right) & \cos \left(k_{z j} \cdot d_{j}\right)
\end{array}\right]
\end{array}
$$

Which combines $e$ and $h$ on both sides of the $j^{\text {th }}$ layer as input and output quantities. The matrix $\overline{\mathrm{T}}_{j}$ is the matrix representation of the $j^{\text {th }}$ layer in the (TM, TE) representation. The boundary conditions for the considered structure presented by (fig.1) in the spectral domain

$$
\overline{\mathbf{e}}_{1}\left(k_{s}, z_{0}^{+}\right)=\overline{0}
$$

$$
\begin{aligned}
& {\left[\begin{array}{l}
\overline{\mathbf{e}}_{2}\left(k_{s}, z_{1}^{+}\right) \\
\overline{\mathbf{h}}_{2}\left(k_{s}, z_{1}^{+}\right)
\end{array}\right]=\bar{T}_{1} \cdot\left[\begin{array}{c}
\overline{\mathbf{e}}_{1}\left(k_{s}, z_{0}^{+}\right) \\
\overline{\mathbf{h}}_{1}\left(k_{s}, z_{0}^{+}\right)
\end{array}\right]-\left[\begin{array}{c}
0 \\
\overline{\mathbf{J}}\left(z_{1}\right)
\end{array}\right]} \\
& \overline{\mathbf{h}}_{2}\left(k_{s}, z_{1}^{+}\right)=\overline{\mathbf{g}}_{0}\left(k_{s}\right) \cdot \overline{\mathbf{e}}_{2}\left(k_{s}, z_{1}^{+}\right)
\end{aligned}
$$

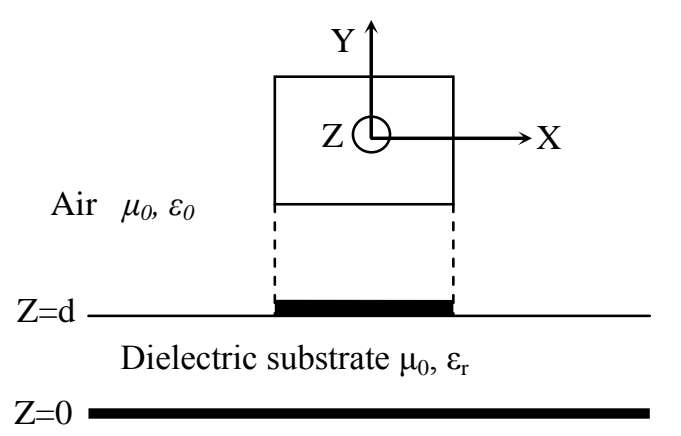

Fig. 1 .Geometrical structure of a rectangular microstrip patch.

The transformed components of the tangential electric field are expressed as function of the transformed current density components on the patch, as

$$
\left[\begin{array}{c}
\tilde{E}_{x} \\
\tilde{E}_{y}
\end{array}\right]=\left[\begin{array}{ll}
G_{x x} & G_{x y} \\
G_{y x} & G_{y y}
\end{array}\right] \cdot\left[\begin{array}{l}
\tilde{J}_{x} \\
\tilde{J}_{y}
\end{array}\right]
$$

The Galerkin moment method is implemented in the Fourier transform domain to reduce the integral equation to a matrix equation. The surface current $\mathbf{J}$ on the patch is expanded into a finite series of known basis functions $J_{x n}$ and $J_{y m}$

With 


$$
\mathbf{J}=\sum_{n=1}^{N} a_{n}\left[\begin{array}{c}
J_{x n} \\
0
\end{array}\right]+\sum_{m=1}^{M} a_{n}\left[\begin{array}{c}
0 \\
J_{y m}
\end{array}\right]
$$

Where $n$ and $m$ are the mode expansion coefficients to be sought. Substituting the vector Fourier transforms. Next, the resulting equation is tested by the same set of basis functions that was used in the expansion of the patch current. Thus, the integral equation is discredited into the following matrix equation:

$$
\begin{aligned}
{\left[\begin{array}{cc}
\left(\bar{Z}_{k n}^{1}\right)_{N \times N} & \left(\bar{Z}_{k m}^{2}\right)_{N \times M} \\
\left(\bar{Z}_{l m}^{3}\right)_{M \times N} & \left(\bar{Z}_{\mathrm{ln}}^{4}\right)_{M \times M}
\end{array}\right] \cdot\left[\begin{array}{l}
\left(a_{n}\right)_{N \times 1} \\
\left(b_{n}\right)_{M \times 1}
\end{array}\right]=\left[\begin{array}{c}
\overline{0} \\
\overline{0}
\end{array}\right] } \\
Z_{k n}^{1}=\int_{-\infty}^{+\infty} \int_{-\infty}^{+\infty} \tilde{J}_{x k}\left(-k_{x},-k_{y}\right) \cdot G_{x x}, \tilde{J}_{x m}\left(k_{x}, k_{y}\right) d k_{x} d k_{y} \\
Z_{k m}^{2}=\int_{-\infty}^{+\infty} \int_{-\infty}^{+\infty} \tilde{J}_{x k}\left(-k_{x},-k_{y}\right) \cdot G_{x y} \tilde{J}_{y m}\left(k_{x}, k_{y}\right) d k_{x} d k_{y} \\
Z_{l m}^{3}=\int_{-\infty}^{+\infty} \int_{-\infty}^{+\infty} \tilde{J}_{y l}\left(-k_{x},-k_{y}\right) \cdot G_{y x} \tilde{J}_{x m}\left(k_{x}, k_{y}\right) d k_{x} d k_{y} \\
Z_{l \mathrm{ln}}^{4}=\int_{-\infty}^{+\infty} \int_{-\infty}^{+\infty} \tilde{J}_{y l}\left(-k_{x},-k_{y}\right) \cdot G_{y y}, \tilde{J}_{y m}\left(k_{x}, k_{y}\right) d k_{x} d k_{y}
\end{aligned}
$$

The system of linear equations given in Eq. 14 has non-trivial solutions when

$$
\operatorname{det}[\mathrm{Z}(\omega)]=0
$$

Equation 15 is an eigen equation for $\omega$, from which the characteristics of the stacked structure of Figure 1 can be obtained. In fact, let $\omega=2 \pi\left(f_{r}+\mathrm{i} f_{i}\right)$ be the complex root of Eq. 15 . In that case, the quantity $f_{r}$ stands for the resonant frequency, the quantity BW $=2 f_{i} / f_{r}$ stands for the bandwidth. In our case eq. 15 represent the fitness function that will be optimized by using PSO algorithm.

\section{PARTICLE SWARM OPTIMIZATION}

The Particle Swarm Optimization is an evolutionary optimization technique, which is a local to global search method based on particle search. It follows the optimization process by means of local best (pbest), global best (gbest), particle displacement and particle velocity. In this paper, all these features have been applied on the determination of the resonant frequency $\left(f_{\mathrm{r}}\right)$ and bandwidth (BW), function of geometric parameters and dielectric constants of rectangular microstrip antenna. The global function approximation capability and generalization capability of Particle Swarm Optimization in the modeling of microstrip antenna have also been studied. This method is used for particles from landing on any solution instead of just the best solution. This is where the social aspect of mind and intelligence comes into play. The particles are considered to move through co-ordinates of $\mathrm{N}$-dimensional space. When particle moves, it sends its co-ordinates to a function and measures its "fitness" value, close to a best solution for the problem. The evolutions of particles, guided only by the best solution, tend to be regulated by behavior of the neighbors. In the simplest form, the position Sand velocity ' $v$ ' of each particle are represented by the following equations considering pbest rather than gbest as the best position of the particle referred to the neighbors. The particle velocity is expressed as [7].

$$
\begin{gathered}
v_{i}(k)=w v_{i}(k-1)+C_{1} r_{1} \times\left(\text { pbest }_{i}-S_{i}(k-1)\right)+ \\
C_{2} r_{2} \times\left(\text { gbest }-S_{i}(k-1)\right)
\end{gathered}
$$

where, $v i(\mathrm{k})$ : velocity of agent $\mathrm{i}$ at iteration $\mathrm{k}$, $\mathrm{w}$ : weighting function, $C_{l}, C_{2}$ : weighting factor, $r_{1}, r_{2}:$ random number between 0 and 1 ,

$S_{i}(\mathrm{k})$ : current position of agent $\mathrm{i}$ at iteration $\mathrm{k}$, pbest $_{i}$ : pbest of agent $i$, gbest : gbest of the group.

Using the above equation, a certain velocity which gradually gets close to pbest and gbest can be calculated. The current position (searching point in the solution space) can be modified by the following equation:

$S_{i}(k)=S_{i}(k-1)+v_{i}(k)$

The basic program flow of PSO is depicted in a flowchart as shown in Fig. 2. As mentioned, the objective of the optimization is the optimization of the matrix impedance $(Z)$ in Eq. 15. 


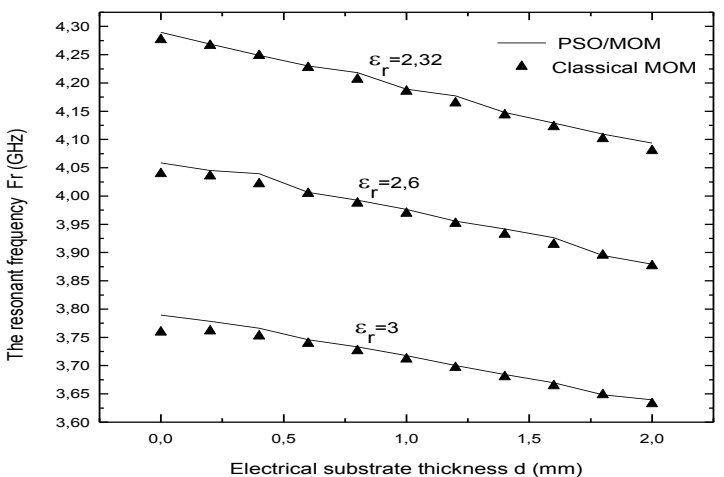

Fig. 3. The resonant frequency versus electrical substrate thickness.

substrates are depicted in Fig. 3. It is evident from Fig 3 that the resonant frequency indeed decreases as the antennas become electrically thicker as has been shown in previously published results in literature [9]

rectangular MSAs are listed and compared with measured results in Table I. The results of this method are in very good agreement with measurements. It can be very useful for the development of fast CAD algorithms.

Fig. 3 and Figure 4 shows the resonant frequency and bandwidth as a function of substrate thickness for different values of dielectric constant $\varepsilon_{\mathrm{r}}=2.32,2.6$ and 3 of a rectangular patch antenna with dimensions $a=1.9 \mathrm{~cm}, b=2.29 \mathrm{~cm}$. From this graphs we see that the results obtained by PSO/MOM algorithms have the same behavior as those obtained by the method of moment.

It should be noted, that the time necessary for calculates parameters of the antenna by PSO/MOM is approximately $50 \mathrm{~s}$ but for traditional moment method is between 5 and $20 \mathrm{mn}$.

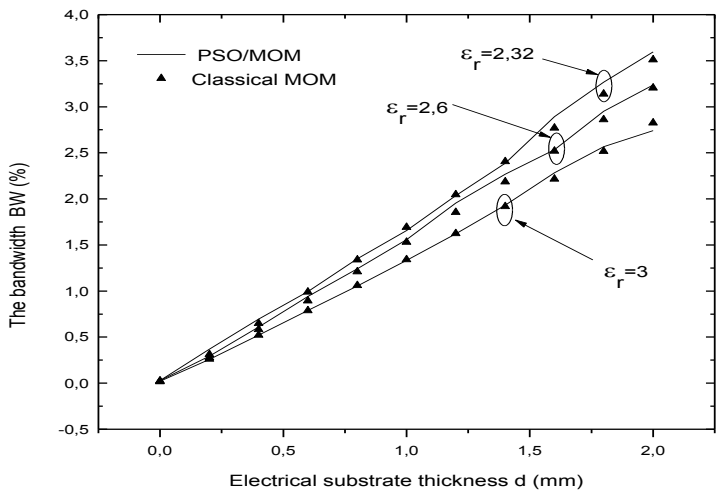

Fig. 4. The bandwidth versus electrical substrate thickness.

\section{CONCLUSIONS}

In this paper, an efficient method for the integration of Particle Swarm Optimization (PSO) with the method of moments (MOM) for microstrip antenna modeling has been presented. PSO/MOM was applied successfully for determination of resonant frequency and bandwidth of a rectangular patch antenna. The calculated results have been compared with measured one available in the literature and excellent agreement has been found. Better accuracy with respect to the previous conventional methods and natural selection method (like PSO) is obtained. Since the method presented in this paper have good accuracy, require no tremendous computational effort, they can be very useful for the development of fast CAD algorithms.

\section{REFERENCES}

[1] D. K. Neag, S. Pattnaik, D.C. Panda, S.Devi, B.Khuntia and M. Dutta, "Design of a wideband microstrip antenna and the use of artificial neural networks in parameter calculation". IEEE Transactions on Antennas and Propagation, VOL. 45, NO. 3, JUNE 2005.

[2] S. Selleri, M. Mussetta, P. Pirinoli, R. E. Zich, , and L. Matekovits, "Some Insight Over New Variations of the Particle Swarm Optimization Method", IEEE Antennas And Wireless Propagation Letters, VOL. 5, 2006.

[3] K. L. Chung and W. Y. Tam; "Particle Swarm Optimization of Wideband Patch Antennas" Microwave Conference, 2008. APMC 2008. Asia-Pacific; 16-20 Dec. 2008

[4] J.S. Dahele and K.F. Lee, "Theory and experiment on microstrip antennas with air gap", IEE Proc Pt H 132 (1985), 455-460.

[5] D. Guha, "Resonant frequency of circular microstrip antennas with and without air gaps", IEEE Trans on Antennas and Propagat 49 (2001), 55-59.

[6] D. Guha, "Resonant frequency of circular microstrip antennas with and without air gaps", IEEE Trans Antennas Propag 49 (2001), 55-59.

[7] V. S. Chintakindi, , S. S. Pattnaik, , O.P.Bajpai, S.Devi, "Resonant Frequency of Equilateral Triangular Microstrip Patch Antenna Using Particle Swarm Optimization Technique", Proceedings of International Conference on Microwave -2008

[8] Y. Shi and Eberhart, "R.CA modified particle swarm optimizer", Proceedings of the IEEE International Conference on Evolutionary Computation., IEEE Press, Piscataway, NJ, 1998, pp.69-73

[9] T. Fortaki, D. Khedrouche, F. Bouttot, A. Benghalia, "A numerically efficient full-wave analysis of a tunable rectangular microstrip patch", INT. J. Electronics, Vol. 91, No 1, (2004). 57-70.

[10] E.Chang, Long S.A., Richards W.F., "An experimental investigation of electrically thick rectangular microstrip antennas", IEEE Trans. Antennas propagate. 34 (1986), 767-772.

[11] K.R., Carver "Practical analytical techniques for the microstrip antenna", Proc. Workshop on printed Circuit antenna technology, New Mexico State University, Las Cruces, ( Oct 1979), 7,1-7,20.

[12] M. Kara. "The resonant frequency of rectangular microstrip antenna elements with various substrate thicknesses". Microwave and Optical Technology Lett, 11(1996). 55-59.

[13] M. Kara. "Closed-form expressions for the resonant frequicy of rectangular microstrip antenna elements with thick substrates". Microwave and Optical Technology Lett. 12 (1996). 131-136. 